Draft version August 20, 2021

Preprint typeset using LATEX style emulateapj v. 08/22/09

\title{
ENERGETICS OF X-RAY CAVITIES AND RADIO LOBES IN GALAXY CLUSTERS
}

\author{
William G. MATHEWs ${ }^{1}$ AND FABRIZIO BRIGHenti ${ }^{1,2}$ \\ Draft version August 20, 2021
}

\begin{abstract}
We describe the formation and evolution of X-ray cavities in the hot gas of galaxy clusters. The cavities are formed only with relativistic cosmic rays that eventually diffuse into the surrounding gas. We explore the evolution of cavities formed with a wide range of diffusion rates but which are otherwise similar. In previous numerical simulations, in which cavities are formed by injecting ultra-hot but non-relativistic gas, cavity formation contributes thermal energy that may offset radiative losses in the gas, thereby helping to solve the cooling flow problem. Contrary to these results, we find that X-ray cavities formed solely from cosmic rays have a global cooling effect. Most cosmic rays in our cavity evolutions do not move beyond the cooling radius and, as the cluster gas is displaced, contribute to a global expansion of the cluster gas. As cosmic rays diffuse away from the cavities, the nearby gas becomes buoyant, resulting in a significant outward mass transfer within the cooling radius, carrying relatively low entropy gas containing cosmic rays to outer regions in the cluster where it remains for times exceeding the local cooling time in the hot gas. This post-cavity mass outflow due to cosmic ray buoyancy may contribute significantly toward solving the cooling flow problem. For example the mass inflow in the Virgo cluster due to radiative cooling can be balanced by buoyant outflow if only a fraction $\sim 0.0005$ of the accretion energy onto the central black hole inflates X-ray cavities with cosmic rays. We describe the energetics, size, stability and buoyant rise of X-ray cavities in detail, showing how each depends on the rate of cosmic ray diffusion.
\end{abstract}

Subject headings: X-rays: galaxies - galaxies: clusters: general - X-rays: galaxies: clusters - galaxies: cooling flows

\section{INTRODUCTION}

The hot gas in galaxy clusters loses energy by X-ray emission but does not cool to low temperatures. In recent attempts to solve this cooling flow problem it has been almost exclusively assumed that feedback energy from accretion onto cluster-centered massive black holes can be delivered to the hot gas in a manner that maintains the observed temperature and density profiles in spite of radiation losses. The solution to this problem is not straightforward. Even when heat is supplied to the cluster gas in an ad hoc, optimized, ideally finetuned manner, either concentrated or distributed over many tens of kiloparsecs, it is found that the temperature and density profiles deviate strongly from those observed (Brighenti \& Mathews 2002; 2003). For example, highly idealized flows in which radiative cooling is perfectly balanced by local heating at every radius are inconsistent with the secular increase in gas density associated with stellar mass loss in the cluster-centered galaxy. In most galaxy groups and clusters a positive temperature gradient $(d T / d r>0)$ is observed in the inner regions. Since the coolest gas is closest to the central source of AGN heating, this central gas must be heated with exquisite precision (on short timescales) to maintain the low temperatures observed.

In spite of these difficulties, a variety of heating mechanisms continue to be investigated to understand the energetics of hot cluster gas. Heating is usually assumed to be associated with the formation of X-ray cavities by jet

\footnotetext{
${ }^{1} \mathrm{UCO} /$ Lick Observatory, Dept. of Astronomy and Astrophysics, University of California, Santa Cruz, CA 95064

2 Dipartimento di Astronomia, Università di Bologna, via Ranzani 1, Bologna 40127, Italy
}

feedback energy proceeding from the central black hole. Weak shock waves emerge when the cluster gas is displaced as cavities form (see McNamara \& Nulsen 2007 for a review). Weak shocks, observed in a few clusters (e.g. Perseus: Fabian et al 2003; Virgo: Forman et al. 2005; 2007), have the desirable capability of dissipating AGN feedback energy over large regions of the cluster gas, as emphasized by Fabian et al (2003). However, energy dissipation by outwardly propagating shock or sound waves is disproportionally concentrated in the central regions of clusters where the gas density gradient is smallest. Over time the cumulative wave dissipation in this central gas (through which all the waves must pass) causes its temperature to become hotter than observed in any cluster (Fujita \& Suzuki 2005; Mathews, Faltenbacher \& Brighenti 2006). It may be possible to forestall cooling in a limited region of a particular cluster with a proper choice of shock Mach numbers (or sound wave frequencies and amplitudes), but this level of fine-tuning seems contrived.

$\mathrm{X}$-ray cavities are thought to provide a convenient measure of the amount of feedback power delivered to the hot gas. Cavities are observed in $\sim 20-25 \%$ of X-ray bright clusters (Birzan et al. 2004; Rafferty et al. 2006), but the incidence of cavities increases as the cooling time of the central gas decreases (Dunn \& Fabian 2006). The work done in displacing a volume $V$ of cluster gas at pressure $P$ is $P V$ and the energy of the material inside the cavity is $P V /(\gamma-1)$. This corresponds to a total cavity energy of $E_{c a v}=[\gamma /(\gamma-1)] P V=4 P V$ where $\gamma=4 / 3$ is often chosen because many (young) cavities are filled with radio synchrotron emission from relativistic electrons and there is little or no observable evidence for hot thermal 
gas inside cavities (e.g. McNamara \& Nulsen 2007).

Assuming that the local buoyancy time $t_{\text {buoy }}$ in the cluster gas is a measure of the cavity lifetime, Rafferty et al. (2006) estimate the "cavity jet power"

$$
P_{c a v}=\frac{E_{c a v}}{t_{b u o y}} .
$$

Rafferty et al. find that $P_{\text {cav }}$ exceeds the total X-ray luminosity $L_{x}$ within the cooling radius (defined where the cooling time is 7.7 Gyrs) in about $60 \%$ of their sample clusters with cavities. They suggest that clusters with $P_{\text {cav }}<L_{x}$ - and the many clusters with no known cavities - are in a phase of their feedback energy cycle that is just now recovering from a recent heating episode. Rafferty et al. suggest that all the energy within the cavities $P V /(\gamma-1)$ may be available to heat the cluster gas, not just the $P V$ work done by the expanding cavity. This implies that the (thermal) energy of material within the cavity is ultimately shared with the cluster gas. Furthermore, for those clusters in which the total cavity jet power is favorable $\left(P_{\text {cav }}>L_{x}\right)$, it is assumed that this energy is distributed throughout the cluster gas in a manner that preserves the characteristic gas temperature and density profiles observed in galaxy clusters. Implicit in this assumed energy distribution is the requirement that the cavity-forming energy is delivered to the cluster gas in an approximately isotropic manner relative to the central black hole.

Another less often considered possible resolution to the cooling flow problem is the hypothesis that gas is only heated at or near the central black hole and then is buoyantly transported far out into the cluster gas, i.e. a circulation flow (Mathews et al. 2003; 2004). One of the initial motivations for mass circulation was the two-temperature (and therefore buoyant) flow observed in the galaxy group NGC 5044 (Buote et al. 2003). However, Temi, Brighenti \& Mathews (2007a,b) have recently found far-infrared emission from dust extending out to $5-10 \mathrm{kpc}$ around many group-centered, X-ray luminous elliptical galaxies. Since the dust lifetime to sputtering destruction in the hot gas is only $\sim 10^{7} \mathrm{yrs}$, this observation provides additional strong evidence for ongoing buoyant outflow from dust-rich cores in the central galaxies. Additional support for buoyant outflow is provided by the large regions of iron enrichment in the hot gas surrounding cluster-centered elliptical galaxies (De Grandi et al. 2004). Since the $50-100 \mathrm{kpc}$ size of these regions enriched by Type Ia supernovae greatly exceeds that of the central galaxy where they occur, an outward mass transfer is essential for their formation. Buoyant outflow is desirable since it can also preserve the observed gas density and temperature profiles (Mathews et al. 2004). Alternatively, these profiles can be preserved if mass circulates outward in momentum-driven, mass-carrying jets (Brighenti \& Mathews 2006).

In most theoretical studies of X-ray cavity evolution, it is assumed that the cavities are inflated with ultrahot gas (occasionally but not always with $\gamma=4 / 3$ ) (e.g. Brüggen \& Kaiser 2002; Reynolds et al. 2005; Gardini 2007; Pavlovski et al. 2007). If this heated gas is transported in jets from the central black hole, then this type of solution implicitly requires an outward mass circulation which in some simulations can be quite large.
For example, in the recent double jet 3D calculation of Heinz et al. (2006), jets with power $W_{j}=10^{46}$ ergs $\mathrm{s}^{-1}$ enter the cluster gas from the origin with velocity $v_{j}=30,000 \mathrm{~km} \mathrm{~s}^{-1}$. This translates into a mass flux of $\dot{M}_{j}=2 W / v^{2}=35 M_{\odot} \mathrm{yr}^{-1}$ and a total injected mass of $M_{j}=3.5 \times 10^{9} M_{\odot}$ over their $10^{8}$ year computation. If ambient cluster gas is entrained in the jets, the outflowing mass could be increased further.

The jet mass flux may be very much less if most of their energy density is in the form of cosmic rays. While it is possible that jets do transport substantial masses of gas from the center (as in Brighenti \& Mathews 2006), we explore here the energetics in the limiting case in which $\mathrm{X}$-ray cavities are formed only with relativistic cosmic rays that can also diffuse into the cluster gas. For simplicity we assume that most of the cosmic rays in cavities arrive in jets and are not produced in local shocks. In this limit the cavities are formed with pure energy with no appreciable component of rest mass or momentum as in previous numerical simulations. Because of their diffusive nature, cosmic rays can eventually penetrate the cluster gas and contribute to the local pressure support. As a consequence the local cluster gas density is reduced by cosmic rays and becomes buoyant. Inhomogeneously distributed cosmic rays are a natural driver of buoyant mass outflow. Gas carried out by cosmic ray buoyancy may never return to the cluster center.

However, we find that few cosmic rays move beyond the cooling radius, so the cluster gas expands and cools globally. Although some heating is expected from shock waves, this heating is offset by the global cooling. Consequently, X-ray cavities containing cosmic rays result in a net cooling of the cluster gas, not heating as generally assumed. Buoyant mass outflow resulting from inhomogeneous cosmic rays and global expansion of the cluster gas may help to resolve the cooling flow puzzle.

Although it is generally believed that X-ray cavities are formed with cosmic rays, this conjecture has only recently been tested with detailed calculations (Mathews \& Brighenti 2007a,b). However, spherical steady state cluster flows including cosmic rays (e.g. Böhringer \& Morfill 1988; Loewenstein et al. 1991) have been developed to explore the possible large scale dynamical influence of cosmic ray pressure gradients and the dissipation of their energy into the thermal gas.

For simplicity in this initial treatment of the global time-dependent energetics of cluster cavity formation with cosmic rays, we do not specify the physical nature of the relativistic fluid (electrons or protons) nor do we calculate radio or inverse Compton fluxes/luminosities which would require additional assumed parameters these important details will be considered in future papers. Nevertheless, the spatial distribution of cosmic rays we calculate defines the region where radio emission could be expected. Since we do not consider Coulomb heating, the cluster gas responds adiabatically to cosmic ray pressure gradients.

\section{EQUATIONS AND COMPUTATIONAL PROCEDURE}

The combined Eulerian evolution of (relativistic) cosmic rays (CRs) and thermal gas can be described with 
the following four equations:

$$
\begin{gathered}
\frac{\partial \rho}{\partial t}+\nabla \cdot \rho \mathbf{u}=0 \\
\rho\left(\frac{\partial \mathbf{u}}{\partial t}+(\mathbf{u} \cdot \nabla) \mathbf{u}\right)=-\nabla\left(P+P_{c}\right)-\rho \mathbf{g} \\
\frac{\partial e}{\partial t}+\nabla \cdot \mathbf{u} e=-P(\nabla \cdot \mathbf{u}) \\
\frac{\partial e_{c}}{\partial t}+\nabla \cdot \mathbf{u} e_{c}=-P_{c}(\nabla \cdot \mathbf{u})+\nabla \cdot\left(\kappa \nabla e_{c}\right)+\dot{S}_{c}
\end{gathered}
$$

where we suppress artificial viscosity terms. Pressures and thermal energy densities in the plasma and cosmic rays are related respectively by $P=(\gamma-1) e$ and $P_{c}=$ $\left(\gamma_{c}-1\right) e_{c}$ where we assume $\gamma=5 / 3$ and $\gamma_{c}=4 / 3$. The cosmic ray dynamics are described by $e_{c}$, the integrated energy density over the cosmic ray energy or momentum distribution, $e_{c} \propto \int E N(E) d E \propto \int p^{4} f(p)\left(1+p^{2}\right)^{-1 / 2} d p$.

The first three equations are the usual equations for conservation of mass, momentum and thermal energy in the hot thermal cluster gas. We do not include optically thin radiative losses since our intention here is to study the energetics of cavity creation alone without the complicating effects of a secular radiative energy loss and central cooling. Note that the CR pressure gradient in equation 2 contributes to the motion of the thermal gas. This exchange of momentum between CRs and gas arises as the CRs diffuse through magnetic irregularities (Alfven waves) that are nearly frozen into the hot thermal gas. However, magnetic terms do not explicitly enter in the equations because typical magnetic fields in cluster gas $\sim 1-10 \mu \mathrm{G}$ (Govoni \& Feretti 2004) are too small, i.e. the magnetic energy densities $\sim B^{2} / 8 \pi \lesssim 10^{-11}$ erg $\mathrm{cm}^{-3}$ are generally much less than the thermal energy density in the hot gas. In addition, the Alfven velocity $v_{A}=B /(4 \pi \rho)^{1 / 2}=2 n_{e}^{-1 / 2} B(\mu \mathrm{G}) \mathrm{km} \mathrm{s}^{-1}$ is typically much less than the sound or flow speeds in cluster gas so the Alfven velocity of the magnetic scatterers can be ignored (e.g. Drury \& Falle 1986, Jones \& Kang, 1990).

Equation 4 above describes both the advection of CRs with the gas and their diffusion through the gas. A mass conservation equation for the CRs is unnecessary because of their negligible rest mass. The $\mathrm{CR}$ diffusion coefficient $\kappa$ is difficult or impossible to calculate in the absence of detailed information about the magnetic field topology which is currently unknown. However, we expect that $\kappa$ may vary inversely with the density of the thermal gas, assuming that the magnetic field strength also scales with density. For simplicity we ignore for now any dependence of $\kappa$ on CR particle momentum. Since observed radio lobes are very approximately spherical, we assume that $\kappa$ is isotropic, consistent with a highly irregular global magnetic field. For these preliminary calculations it is not necessary to specify the CR composition, either electrons or protons can dominate as long as they are relativistic. Finally, we assume that the total CR energy density is not substantially reduced by losses due to synchrotron emission or interactions with ambient photons or thermal particles during the cavity evolution time. Dissipation of cosmic ray energy into the thermal gas is probably not important in X-ray cavities since the gas temperature in the cavity rims is in fact relatively cooler.

The set of equations above are solved in $(r, z)$ cylindrical coordinates using a $2 \mathrm{D}$ code very similar to ZEUS 2D (Stone \& Norman 1992). To be specific, we study the evolution of X-ray cavities in the well-observed Virgo cluster using the analytic fits to the observed gas temperature and density profiles suggested by Ghizzardi et al. (2004). The computational grid consists of 100 equally spaced zones in both coordinates out to $50 \mathrm{kpc}$ plus an additional 100 zones in both coordinates that increase in size logarithmically out to $\sim 1 \mathrm{Mpc}$. We adopt reflection boundary conditions for gas and cosmic rays at the origin and, in view of the large computational domain, also at the outer grid boundary. We assume a spherical gravitational field $\mathbf{g}=\left(g_{z}, g_{r}\right)$ that establishes exact initial hydrostatic equilibrium for the Virgo cluster gas pressure gradient. The cosmic ray diffusion term in equation 4 is solved using implicit Crank-Nicolson differencing. While this differencing scheme is stable for all time steps, we restrict each time step by the stability condition for explicitly-differenced diffusion as well as the Courant condition for numerical stability. Shocks are treated with a standard artificial viscosity.

We assume that the X-ray cavity is formed by CRs that propagate in a non-thermal jet from the central black hole (AGN) to some fixed radius. The CRs are deposited in a gaussian-shaped sphere of characteristic radius $r_{s}=$ $2 \mathrm{kpc}$ located at $\mathbf{r}_{c a v}=(r, z)=(0,10 \mathrm{kpc})$, i.e. $10 \mathrm{kpc}$ along the $z$-axis. The $\mathrm{CR}$ source term in equation 4 is therefore

$$
\dot{S}_{c}=\frac{E_{c a v}}{t_{c a v}} \frac{e^{-\left(\left(\mathbf{r}-\mathbf{r}_{c a v}\right) / r_{s}\right)^{2}}}{\pi^{3 / 2} r_{s}{ }^{3}} \quad \operatorname{erg~cm}^{-3} \mathrm{~s}^{-1}
$$

when $t<t_{c a v}$. The integral of $\left(r_{s} \pi^{1 / 2}\right)^{-3} e^{-\left(r / r_{s}\right)^{2}}$ over space is unity.

In the calculations described below our principal objective is to explore the unique diffusive effects of cosmic rays on cavity formation and energetics. We therefore restrict the total $\mathrm{CR}$ energy of all calculations to $E_{c a v}=1 \times 10^{58}$ ergs and adopt $t_{c a v}=2 \times 10^{7}$ yrs as the $\mathrm{CR}$ injection time. We choose $t_{c a v}$ to be consistent with X-ray cavity observations; it is shorter than the local buoyancy time in the cluster gas but sufficiently long not to produce strong shocks which are not commonly observed. At times $t>t_{\text {cav }}$ when $\dot{S}_{c}=0$, the total CR energy $E_{c r}=\int e_{c} d V$ over the grid volume remains very nearly constant but changes slightly due to advection in adiabatic compressions or rarefactions.

Since the CR diffusion coefficient is poorly known at present - and may vary from one AGN event to another - we consider a wide range of density-dependent coefficients,

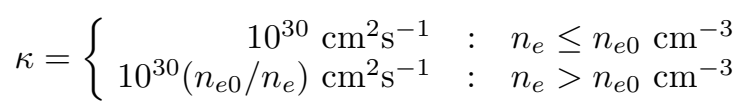

In general, for reasons discussed above, we assume that the CR diffusion $\kappa$ varies inversely with cluster gas density. But $\kappa$ must be sufficiently large so that the CR density is approximately uniform in the $\mathrm{X}$-ray cavities where the cluster gas density is lowest, i.e. we assume that CRs also diffuse inside the cavity. For example when $E_{c a v}=1 \times 10^{58}$ ergs we find that the maximum cavity 
radius is $\sim 5 \mathrm{kpc}$ so we require that $\kappa$ in the cavities be at least $(5 \mathrm{kpc})^{2} / \mathrm{t}_{\mathrm{cav}} \approx 4 \times 10^{29} \mathrm{~cm}^{2} \mathrm{~s}^{-1}$ and this condition is ensured by our adopted $\kappa\left(n_{e}\right)$ above. In regions of higher $n_{e}$ the density parameter $n_{e 0}$ determines the $\mathrm{CR}$ diffusion coefficient. In the following we consider $n_{e 0}=6 \times 10^{-3}-6 \times 10^{-6} \mathrm{~cm}^{-3}$. The largest CR diffusion coefficient $\kappa$ (corresponding to $n_{e 0}=6 \times 10^{-3}$ ) is similar to that required by Mathews \& Brighenti (2007b) to explain a common age $\left(10^{8}\right.$ yrs $)$ for the large radio lobes and the cavity jet (thermal filament) observed by Forman et al. (2007) in the Virgo cluster.

\section{CAVITY EVOLUTION WITH DIFFERENT COSMIC RAY DIFFUSIVITIES}

Figure 1 shows the gas density $\rho(r, z)$ and cosmic ray energy density $e_{c}(r, z)$ at six times during the evolution of an X-ray cavity and its cosmic ray (radio) lobe. In Figure $1 n_{e 0}=6 \times 10^{-3} \mathrm{~cm}^{-3}$ so the diffusion coefficient is rather large, but identical to that used by Mathews \& Brighenti (2007b) to describe the evolution of the cavity jet (thermal filament) in M87/Virgo. The cavity in Figure 1 is formed $10 \mathrm{kpc}$ along the (horizontal) zaxis during time $t_{\text {cav }}=0.02$ Gyr. At time 0.024 Gyr, shortly following $t_{c a v}$, the cosmic rays (dotted contours) are tightly confined inside the cavity. By time 0.066 Gyr the cosmic rays have diffused through the cavity walls forming a small radio lobe and the cavity has just disappeared - we define the cavity as that region where the plasma density is lower than the original Virgo density by at least a factor $1 / 3$. A small vortex at $(r, z)=(5,20)$ $\mathrm{kpc}$ is visible at this time. During the first three times shown in Figure 1 a (relatively cooler) thermal feature (the "cavity jet") is seen to rise along the $z$-axis. The evolution of this feature is described in detail in Mathews \& Brighenti (2007b) and we will not repeat that discussion again here. During the three later times in Figure 1 the denser parts of the cavity jet have fallen back toward the center of Virgo and the hot gas dynamics become more quiescent. However, the cosmic rays are seen to diffuse into a progressively larger region elongated along the $z$-axis. Although not apparent in the gas density contours, there is a net outward buoyant migration of the gas in the region occupied by the cosmic rays (the radio lobe).

Figure 2 shows the cavity evolution when the cosmic ray diffusion is 1000 times lower (when $n_{e}>n_{e 0}=$ $6 \times 10^{-6} \mathrm{~cm}^{-3}$ ) but with all other parameters unchanged. Now the cosmic rays are seen to be very tightly confined to the cavity region until at least time 0.1 Gyr when the cavity is still visible. In this evolution the outer parts of the cavity break away forming a vortex that migrates away from the $z$-axis, carrying its own cosmic rays. By time 0.3 Gyr the brightest parts of the radio image (corresponding to the largest cosmic ray energy density) should consist of two separate regions, a feature along the $z$-axis extending out to $40 \mathrm{kpc}$ and the vortex at $(r, z)=(14,38) \mathrm{kpc}$. At this time $(0.3 \mathrm{Gyr})$ we see an enhanced gas density that accompanies the cosmic rays along the $z$-axis. At still later times the cosmic rays continue to reside mostly along the $z$-axis but by time 0.9 Gyr a region of low cosmic ray energy density becomes visible in the $r$-direction along the trajectory of the receding vortex (now at $(r, z)=(25,28) \mathrm{kpc}$ ).

When X-ray cavities are formed in an atmosphere ini- tially at rest, as we assume here, the vortex region at time 0.3 Gyr in Figure 2 would appear as a ring when viewed along the $z$-axis. To our knowledge no ring-shaped radio features have been observed. This could simply be due to the faintness of such regions since radio-synchrotron electrons tend to not to produce observable $\mathrm{GHz}$ emission after about $10^{8}$ yrs. Nevertheless, it is clear from Figures 1 and 2 that the radio lobe morphology can in principle provide valuable information concerning the cosmic ray diffusion coefficient about which very little is known at present. Finally, since gas phase metal abundances tend to increase toward the centers of clusters, regions of enhanced abundance are expected to accompany the outward buoyant migration of cosmic rays.

As a further aid in interpreting Figures 1 and 2, Figure 3 shows the pressure profiles along the $z$-axis at four times for both evolutionary calculations. At early times the cavity is visible as the region where $P_{c}>P$. Within the cavity the pressure gradient $\left(\sim d P_{c} / d r\right)$ is nearly flat because of the enormous pressure scale height of the relativistic fluid. However, except near and within the cavities, the total pressure $P+P_{c}$ (dotted lines) deviates very little from the pressure profile in the cluster before the cavity was introduced (dot-dashed lines). This is a consequence of the largely subsonic character of the flows. After about 0.1 Gyrs in both calculations the cosmic rays diffuse sufficiently so that the pressure ratio $P_{c} / P \ll 0.2$, the current threshold of $\gamma$-ray detectability of cosmic ray protons if nearby clusters are completely filled with cosmic rays (Pfrommer \& Ensslin et al. 2004). Although the cosmic ray pressure and energy density are negligible at these late times, we show below that cosmic rays continue to displace about the same volume of gas. This results in a very long-lasting global expansion of cluster gas that can be seen by the small discrepancies in the $z$-axis gas pressure profile (solid lines) relative to that in the initial cluster (dash-dotted lines) at times 0.3 Gyrs in Figure 3. Nevertheless, the close similarity of the initial and final gas pressure along the $z$-axis (and elsewhere) at late times is consistent with our finding that the gas density and temperature (and therefore entropy ${ }^{1}$ ) gradients are also only slightly affected by the cavity evolution.

\section{ENERGETICS AND EVOLUTION OF X-RAY CAVITIES}

Figure 4 shows the evolution of cluster gas energies resulting from cavity-lobe formation with cosmic rays. The four panels show the evolution as the cosmic ray diffusion coefficient $\kappa$ decreases with $n_{e 0}$ (in $\mathrm{cm}^{-3}$ ) over a wide range: $n_{e 0}=6 \times 10^{-3}($ panel $a), 6 \times 10^{-4}$ (panel b), $6 \times 10^{-5}($ panel $c)$, and $6 \times 10^{-6}$ (panel $\left.d\right)$. The total cosmic ray energy integrated over the entire computational region $E_{c r}=\int e_{c} d V$ is shown with long dashed lines. $E_{c r}(t)$ is seen to rise until $t_{c a v}=2 \times 10^{7} \mathrm{yrs}$ as the cavities form, then remain approximately constant after the cosmic ray source is turned off. $E_{c r}$ is not a strictly conserved energy. The small decrease in $E_{c r}(t)$

\footnotetext{
1 Figure 8 shows (for the evolution in Figure 2) an approximate $z$-axis gas entropy profile at 0.9 Gyrs relative to the initial atmosphere. When plotted, $s(z)$ at 0.9 Gyrs is almost identical to that in the original atmosphere from the origin to $z \approx 25 \mathrm{kpc}$ and beyond $z \approx 42 \mathrm{kpc}$. In the intermediate region $25<z<42$ kpc the entropy at 0.9 Gyrs is depressed below the original profile approximately as shown in Figure 8.
} 
visible at times $t>t_{\text {cav }}$ can occur if the cosmic ray energy density is reduced by a secular advective expansion with the cluster gas. This decrease in $E_{c r}(t)$ after $t_{\text {cav }}$ is stronger when $\kappa$ is smaller (panels $a \rightarrow d$ ) since the cosmic rays are more confined near the $z$-axis where most of the gas expansion occurs. The (small) total kinetic energy in the cluster gas $E_{k i n}=0.5 \int \rho\left(u_{x}^{2}+u_{y}^{2}\right) d V$ is shown with dotted lines in each panel. The change in potential energy compared to the initial Virgo atmosphere, $\Delta E_{\text {pot }}=\int \phi[\rho(t=0)-\rho] d V$ is shown with short dashed lines. The gravitational potential $\phi(R)$ is found from the initial M87/Virgo atmosphere by integrating $d \phi / d R=-(1 / \rho) d P / d R$ where $R=\left(r^{2}+z^{2}\right)^{1 / 2}$ is the radial coordinate. The change in the total gas thermal energy relative to the original atmosphere $\Delta E_{t h}=$ $\int[e-e(t=0)] d V$ is shown with (the lower) solid lines in each panel. Finally the dash-dotted line shows the total energy $E_{t o t}=\Delta E_{t h}+\Delta E_{p o t}+E_{k i n}+E_{c r}$ which is constant after time $t=t_{c a v}$ to an excellent approximation and is equal to $E_{c a v}=10^{58}$ ergs as expected.

Also shown in each panel of Figure 4 is an approximate evaluation of the quantity $4 P V$ where $V$ is the volume of the X-ray cavity at any time (arbitrarily defined as the sum over all grid zone volumes in which the gas density is less than $\rho(t=0) / 3)$ and $P$ is an estimate of the average pressure in the (sometimes noncontiguous) zones containing the cavity. When cosmic rays are strongly trapped within the cavity, we expect $4 P V=E_{c a v}=E_{t o t}$. In panel $a$ of Figure 4 we see that $4 P V<E_{\text {cav }}$ which can be expected for larger $\kappa$ when cosmic rays diffuse through the cavity walls. However, $4 P V>E_{c a v}$ is apparent in panels $c$ and $d$, although $4 P V$ never exceeds $E_{\text {cav }}=10^{58}$ ergs by more than about $20 \%$. This may be due to our rather approximate estimate of $P$ and $V$ or it may be a real inertial overshoot just after the cavity is formed. In any case, we include $4 P V(t)$ in Figure 4 because this is the cluster gas heating energy proposed by Rafferty et al. (2006) and McNamara \& Nulsen (2007) in their discussions of cavity energetics.

Figure 5 shows the approximate evolution of the cavity radius $r_{c a v}(t)$ and its mean radius in the cluster $R_{\text {cav }}(t)$. The cavity radius is found from the estimated cavity volume $V$ by assuming that the cavity is spherical, $r_{c a v}=(3 V / 4 \pi)^{1 / 3}$. The four lines for $r_{c a v}$ and $R_{c a v}$ in Figure 5 correspond to the four cosmic ray diffusivities $\kappa$ as it decreases in each panel in Figure 4: $a$, dotted line; $b$, short dashed line; $c$, long dashed line; $d$, solid line. The buoyant trajectory of the cavities $R_{\text {cav }}(t)$ is independent of $\kappa$, but the cavities progress further along $R_{\text {cav }}(t)$ when $\kappa$ is smaller. The cavity lifetime is determined when $r_{c a v} \rightarrow 0$. Notice that the cavity lifetimes are similar for $n_{e 0}=6 \times 10^{-5}$ (long dashed line), and $n_{e 0}=6 \times 10^{-6}$ (solid line), suggesting that the cavity evolution would not change much if $n_{e 0}$ (and therefore $\kappa)$ were reduced further.

The main result we wish to emphasize is the evolution of the cluster gas thermal energy $\Delta E_{t h}(t)$. At early times during cavity formation when $t \lesssim t_{c a v}, \Delta E_{t h}$ increases because of heating due to the weak shock that propagates away from the expanding cavity. This initial shock heating increases as $\kappa$ decreases (panels $a \rightarrow d$ in Figure 4) because (for fixed $E_{c a v}$ and $t_{c a v}$ ) the shock strength increases when cosmic rays are more confined within the cavity. This is consistent with the $1 \mathrm{D}$ cavity evolution described by Mathews \& Brighenti (2007a). However, after the cavity is formed $\left(t \gtrsim t_{c a v}\right) \Delta E_{t h}(t)$ decreases and becomes negative after $t \approx 3-5 \times 10^{7}$ yrs. We see in Figure 4 that the final energy separation between $\Delta E_{t h}$ and $\Delta E_{\text {pot }}$ is independent of $\kappa$. Also notice that in each panel of Figure 4 the average final value of the energy change $0.5\left(\Delta E_{t h}+\Delta E_{\text {pot }}\right)$ is not zero, but is approximately equal to the peak thermal energy acquired from the shock, $\sim \Delta E_{t h}\left(t_{c a v}\right)$. Although the total energy change in the cluster $\Delta E_{p o t}+\Delta E_{t h}+E_{k i n}$ increases as $\kappa$ decreases, the cluster gas experiences a net cooling $\left(\Delta E_{t h}<0\right)$ when cavities are formed with cosmic rays. This global cooling is exactly opposite to the results discussed by other authors in which cavity formation is regarded as an important source of AGN heating required to balance radiative losses in the cluster gas and reduce central cooling in cooling flows.

The energy evolutions shown in Figure 4 are not qualitatively altered when the total cavity energy $E_{c a v}$ is increased. For example when $E_{c a v}=10^{59}$ ergs (deposited at $(r, z)=(0,10) \mathrm{kpc}$ in time $t_{c a v}=2 \times 10^{7} \mathrm{yrs}$ with $\left.n_{e 0}=6 \times 10^{-6} \mathrm{~cm}^{-3}\right)$ all energies are larger but the proportions are similar to those in Figure 4. Although the shock is stronger in this case, so is the buoyant cooling and $\Delta E_{t h}$ still becomes negative after $\sim 10^{8}$ yrs. The maximum equivalent spherical radius of this high energy cavity is about $r_{\text {cav }}=15 \mathrm{kpc}$ at $\log t \sim 8.2$, but the cavity disappears soon afterward at $\log t=8.4$ when $R_{\text {cav }}=55 \mathrm{kpc}$. More energetic cavities are bigger and more buoyant, but don't last proportionally longer.

\section{GLOBAL EXPANSION AND MASS OUTFLOW}

To verify that the cluster gas has in fact undergone a net expansion as a result of cavity formation, we computed the evolution of the cumulative gas mass distribution $M(R)$. First we sorted the computational zones in cylindrical coordinates in order of increasing spherical radius $R=\left(r^{2}+z^{2}\right)^{1 / 2}$ then we integrated over the 7855 sorted zones within $R=50 \mathrm{kpc}$ to determine $M(R)$. Figure 6 shows $M(R)$ in (one hemisphere of) the initial cluster $(t=0)$ and after $t=0.9$ Gyr for two limiting values of $\kappa\left(n_{e 0}=6 \times 10^{-3}\right.$ and $\left.6 \times 10^{-6} \mathrm{~cm}^{-3}\right)$. To more clearly illustrate the evolution of $M(R)$ most of the $R$-variation in Figure 6 has been removed by plotting $M(R)(R / 10 \mathrm{kpc})^{-2}$. The noise in these plots of $M(R)$ arises because of the finite number of computational grid zones. Figure 6 clearly shows that mass has been removed from the inner regions of the cluster as a result of cavity formation, i.e. the cluster gas has experienced a net expansion. This expansion is remarkably insensitive to the cosmic ray diffusivity $\kappa$, particularly at $R \lesssim 25$ kpc. The evolution of $M(R)$ is expected to depend both on the cavity energy $E_{c a v}$ and the location of the initial cavity in the cluster gas (10 kpc in our case).

The largest radius plotted in Figure $6, R=50 \mathrm{kpc}$, is essentially the cooling radius for the cluster, i.e. the radius where the local cooling time is 7.4 Gyrs, which is comparable to the cluster age. From this plot we estimate that $\Delta M_{10}=1.14 \times 10^{8} M_{\odot}$ has been removed from within $10 \mathrm{kpc}$ for both values of $n_{e 0}$. At $R=50 \mathrm{kpc}$ the mass outflow is $\Delta M_{50}=3.6 \times 10^{8} M_{\odot}$ (for $\left.n_{e 0}=6 \times 10^{-3}\right)$ and $\Delta M_{50}=5.6 \times 10^{8} M_{\odot}($ for 
$\left.n_{e 0}=6 \times 10^{-6}\right)$. Values of $\Delta M$ refer to the total cluster mass flow in both hemispheres, assuming that a pair of cavities are produced by symmetric double jets.

Using the Ghizzardi et al. (2004) density and temperature profiles for Virgo, we estimate a feedback-free gas cooling rate of $\dot{M}_{c f} \approx 8 M_{\odot} /$ yr. Therefore if cavities similar to the one we calculate here are formed with cosmic rays every $6 \times 10^{7}$ yrs, this could result in a mass outflow at $R=50 \mathrm{kpc}$ equal to the cooling inflow at $R=0$ from radiation losses. When $E_{c a v}=10^{59} \mathrm{ergs}$ the total mass flowing beyond $50 \mathrm{kpc}$ after time 0.9 Gyr is much larger, $\Delta M_{50}=4.6 \times 10^{9} M_{\odot}$. (Note that the buoyant mass outflow scales with $E_{\text {cav }}$, but the cavity lifetime does not.) Successive cavities in Virgo with $E_{\text {cav }}=10^{59}$ erg appearing every $6 \times 10^{8}$ years could balance the mass flow from radiative cooling $\dot{M}_{c f} \approx 8 M_{\odot} /$ yr and also be consistent with the current absence of large cavities in M87/Virgo. Of course an expansion outflow at the cooling radius comparable to the steady state cooling rate $\dot{M}_{c f}$ does not in itself shut down radiative cooling near the cluster center, but the cooling radius will become larger and the cooling time at every radius will increase due to the slightly lower gas density resulting from the global expansion.

\section{WHAT INCREASES THE POTENTIAL ENERGY AND DRIVES THE MASS OUTFLOW?}

The post-cavity mass flow has two aspects: (1) an increase in $\Delta E_{\text {pot }}$ in Figure 4 and non-zero $\Delta M_{50}$ in Figure 6 that result from the global lifting that occurs when a new cavity is formed, and (2) a mass circulation driven near the $z$-axis by buoyant gas containing cosmic rays. We discuss each in turn.

\subsection{Global Lifting}

Although gas certainly flows beyond $R=50 \mathrm{kpc}$ in all our cavity evolutions, the fraction of total cosmic ray energy within this radius $E_{c r, 50} / E_{c r}$ varies. For example, at $t=0.9$ Gyr and large $\kappa\left(n_{e 0}=6 \times 10^{-3} \mathrm{~cm}^{-3}\right)$, the cosmic ray energy in $R<50 \mathrm{kpc}, E_{c r, 50}=66.3 \times$ $10^{56} \mathrm{ergs}$, is less than the total cosmic ray energy on the computational grid, $E_{c r}=81.5 \times 10^{56}$ ergs. In this case about $20 \%$ of the cosmic rays have moved beyond $50 \mathrm{kpc}$. For smaller $\kappa\left(n_{e 0}=6 \times 10^{-3} \mathrm{~cm}^{-6}\right)$ after this same time we find that $E_{c r, 50}=41.8 \times 10^{56}$ ergs is identical to the total energy $E_{c r}$, i.e. almost all cosmic rays are confined within $50 \mathrm{kpc}$. (Both $E_{c r, 50}$ and $E_{c r}$ refer to a single hemisphere.) However, the mass $\Delta M_{50}$ flowing across $R=50 \mathrm{kpc}$ is positive for both cases involving large and small diffusivities $\kappa$.

Evidently mass can flow across the cooling radius even in the absence of local buoyant transport. This must arise because of the quasi-adiabatic expansion of the entire cluster gas when cavities are formed. To understand this better, we begin by showing that the volume of gas displaced by cosmic rays $V_{c}$ depends on the local pressure $P_{a}$ in the cluster atmosphere and the total energy $E_{c r}$ of the cosmic rays, but not on the ratio of cosmic ray to gas partial pressures, $P_{c} / P$, which changes as the cosmic rays diffuse into the gas. Imagine a uniform gas containing a spherical region of volume $V$ that contains a total cosmic ray energy $E_{c r}$. In pressure equilibrium $P_{a}=P_{c}+P$ where $P_{c}=\left(\gamma_{c}-1\right) E_{c r} / V$ and therefore
$V=\left(\gamma_{c}-1\right) E_{c r} / P_{c}$. But the partial volume $V_{c}$ containing gas that is actually displaced by the cosmic rays is $V_{c}=\left(P_{c} / P_{a}\right) V=\left(\gamma_{c}-1\right) E_{c r} / P_{a}$. Therefore as cosmic rays diffuse into larger volumes $V$, the total volume of gas that is displaced $V_{c}$ remains unchanged and independent of $\kappa$. In a non-uniform cluster environment the volume of gas displaced by a given total energy of cosmic rays $E_{c r}$ varies with cluster radius as $P_{a}(R)^{-1}$.

$\Delta M_{50}$ can be estimated by assuming that all cosmic rays in the original cavity do not diffuse beyond their original cavity volume $V_{\text {cav }}$ and remain at their initial cluster radius $R_{c a v}=10 \mathrm{kpc}$. In this limit, and after several cluster sound crossing times, we expect that the mass flow across $R=50 \mathrm{kpc}$ will be equal to the gas mass displaced by the original cavity, $\Delta M_{50} \approx V_{\text {cav }} \rho\left(R_{\text {cav }}\right)=$ $6.2 \times 10^{8} M_{\odot}$ where $V_{\text {cav }}=(4 \pi / 3) r_{\text {cav }}^{3}, r_{\text {cav }} \approx 6 \mathrm{kpc}$ and $\rho\left(R_{\text {cav }}\right)=4.72 \times 10^{-26} \mathrm{gm} \mathrm{s}^{-1}$ is the original cluster density at $R_{\text {cav }}$. This simple estimate of the outflowing mass is very similar to that found from Figure $6, \Delta M_{50}=$ $5.6 \times 10^{8} M_{\odot}$.

However, discrepancies between computed and estimated masses $\Delta M_{50}$ are expected because of several additional details. The cosmic ray energy inside the estimated cavity volume is $E_{c r}=3 P_{10} V_{c a v}=101 \times 10^{56}$ ergs, where $P_{10}=P_{a}(R=10 \mathrm{kpc})=1.28 \times 10^{-10}$ dyne $\mathrm{cm}^{-2}$. However, this energy is about twice as large as the value of $E_{c r}\left(t_{c a v}\right)$ in the low- $\kappa$ evolution shown in panel $d$ of Figure 4 , so the estimated value of $\Delta M_{50}$ above should be reduced by a factor of $\sim 2$. However, $\Delta M_{50}$ is expected to differ from $V_{\text {cav }} \rho\left(R_{\text {cav }}\right)$ because of changes in the mean volume $V_{c}$ of gas displaced by cosmic rays after their outward motion during time 0.9 Gyr. To estimate this suppose that the $e_{c}$-weighted mean radius of cosmic rays at time $0.9 \mathrm{Gyr}$ is $R=30 \mathrm{kpc}$ (see Figure 2 ) where the gas pressure is $P_{30}=0.608 \times 10^{-10}$ dyne $\mathrm{cm}^{-2}$. For constant total cosmic ray energy we expect the volume of gas displaced to vary as $V_{c} \propto P_{a}{ }^{-1}$. Therefore if most of the cosmic rays are transported to $\sim 30$ kpc, our original estimate of $\Delta M_{50}$ must be increased by $\sim P_{10} / P_{30}=2.1$. The combination of both corrections leaves our original estimate nearly unchanged. Considering the uncertainties in these estimates, we conclude that the total mass flow past $R=50 \mathrm{kpc}$ found from Figure 6 for the low $\kappa$ evolution, $\Delta M_{50}=5.6 \times 10^{8} M_{\odot}$, is consistent with a quasi-adiabatic expansion of the Virgo atmosphere past radius $50 \mathrm{kpc}$ due to gas displaced by cosmic rays when the cavity formed and no buoyant mass flow across $R=50 \mathrm{kpc}$ is required.

\subsection{Buoyant Gas Circulation}

In addition to this atmospheric lifting, buoyant mass flow largely within the cooling radius also contributes to the post-cavity cluster evolution. Buoyant gas increases $\Delta E_{\text {pot }}$ and as it expands we expect $\Delta E_{t h}$ to decrease. But every buoyant element must be accompanied elsewhere in the hot gas atmosphere by a downward replacement flow, largely balancing the buoyant changes in $\Delta E_{p o t}$ and $\Delta E_{t h}$. If cavities are created by feedback following a small accretion onto the central AGN, a buoyant mass circulation flow is a natural response to the inflow expected from radiative cooling. The low- $\kappa$ cavity evolution $\left(n_{e 0}=6 \times 10^{-6} \mathrm{~cm}^{-3}\right)$ in Figure 2 is particularly interesting because all buoyant motion occurs within the 
cooling radius at $50 \mathrm{kpc}$.

Figure 7 shows contours of the radial velocity $v_{r}(r, z)$ at four times during the low- $\kappa$ cavity evolution in Figure $2\left(n_{e 0}=6 \times 10^{-6} \mathrm{~cm}^{-3}\right)$. (A plot of the radial velocity in the high- $\kappa$ cavity evolution shown in Figure 1 is almost identical.) We plot $v_{r}(r, z)$ only near the $z$-axis since the radial velocities elsewhere in the atmosphere are very much smaller. Figure 7 shows a strong and sustained subsonic outflow until at least 0.1 Gyr. At $t=0.3 \mathrm{Gyr}$ a backflow occurs near the $z$-axis as the densest parts of the thermal "cavity jet" fall back toward the cluster center (see Mathews \& Brighenti 2007b for more details). Later at $t=0.5 \mathrm{Gyr}$ a mixture of positive and negative radial velocities is visible but all velocities are smaller and this trend toward quiescence continues until $t=1$ Gyr.

Because the entropy decreases monotonically with radius in the initial cluster atmosphere, we can use the entropy as a tracer to confirm that there has been a net mass outflow within $50 \mathrm{kpc}$ during the low- $\kappa$ evolution (which is essentially adiabatic apart from the weak shock wave). We define the gas entropy as $s=10^{-33}\left(e / \rho^{5 / 3}\right)$ in cgs units. In addition we expect that the buoyancy and outward flow of each gas element will increase with the partial pressure of the cosmic rays, $P_{c} /\left(P+P_{c}\right)$. In the upper panel of Figure 8 we plot $P_{c} /\left(P+P_{c}\right)$ against $\Delta s=s(t=0.9)-s_{a t m}$, the change between the entropy $s$ at 0.9 Gyr and the entropy at the same location in the original Virgo atmosphere, $s_{\text {atm }}$. Each point corresponds to a computational grid zone. In those regions where $P_{c} /\left(P+P_{c}\right)$ is largest we see that $\Delta s$ decreases systematically with $P_{c} /\left(P+P_{c}\right)$, indicating that gas containing more cosmic rays at time 0.9 Gyr originally came from high entropy regions closer to the cluster center.

The lower panel of Figure 8 shows (with small points) the location in the cluster at time $0.9 \mathrm{Gyr}$ that contains gas with $P_{c} /\left(P+P_{c}\right)>0.3$ and the open squares mark those (significantly overlapping) zones where $\Delta s<-0.1$ for the low $-\kappa$ cavity evolution. Gas with the highest cosmic ray content is also the gas with the lowest entropy compared to that at the same radius in the original cluster. The spatial distribution of this low entropy gas is similar to the cosmic ray contours in the final panel of Figure 2. Low entropy regions in the lower panel of Figure 8 also have a slightly smaller gas density than adjacent gas at the same cluster radius $R$ without cosmic rays. During times of interest for the cluster evolution, the gas in these post-buoyant regions containing cosmic rays will never return to the cluster core where it initially resided. Figure 8 shows conclusively that low entropy gas originally at small cluster radii has been buoyantly transported outward during the low- $\kappa$ post-cavity evolution, but very little has moved beyond $50 \mathrm{kpc}$ by time $0.9 \mathrm{Gyr}$. Cosmic rays from cavities are driving a mass circulation in the cluster gas out to a large fraction of the cooling radius.

Evidently the long-term decrease in cluster gas mass $\Delta M_{10}=1.14 \times 10^{8} M_{\odot}$ within $R=10 \mathrm{kpc}$ where the cavities formed arises due to the combined effects of the jet of thermal gas along the $z$-axis and the cosmic ray buoyancy that prevents much of this gas from returning back to $R=10 \mathrm{kpc}$. Recall that $\Delta M_{10}$ is nearly independent of the cosmic ray diffusion coefficient. The cosmic ray energy required to remove a solar mass of gas from $R=10 \mathrm{kpc}$ is about $e_{\odot}=E_{\text {cav }} / \Delta M_{10}=8.8 \times 10^{49}$ $\operatorname{erg} M_{\odot}{ }^{-1}$. If $\dot{M} \delta t=8 M_{\odot}$ would cool each year in Virgo due to radiation losses alone, the cosmic ray luminosity required for a buoyant outflow to balance $\dot{M}$ is $L_{c r}=e_{\odot} \dot{M}=7.0 \times 10^{50} \mathrm{erg} \mathrm{yr}^{-1}$ or $2.2 \times 10^{43} \mathrm{erg} \mathrm{s}^{-1}$. However, the rate that energy is created by accretion onto the $\sim 3 \times 10^{9} M_{\odot}$ central black hole in M87/Virgo is $L_{a c c} \approx 0.1 \dot{M} c^{2}=4.6 \times 10^{46} \mathrm{erg} \mathrm{s}^{-1}$. The cosmic ray luminosity $L_{c r}$ required to balance a radiating cooling mass inflow in Virgo is $\sim 2000$ times less than the accretion luminosity generated by the black hole $L_{a c c}$. Therefore, if only a fraction $\epsilon_{c r} \approx 5 \times 10^{-4}$ of the accretion energy converts to cosmic rays that inflate $\mathrm{X}$-ray cavities, this might be sufficient to shut down the cooling flow. A subsequent calculation with multiple cavities and radiative cooling will be required to verify this.

\section{DISCUSSION}

In previous numerical simulations of X-ray cavity evolution (e.g. Brüggen \& Kaiser 2002; Reynolds et al. 2005; Gardini 2007; Pavlovski et al. 2007) as well as in the recent review of McNamara \& Nulsen (2007) it has been concluded that the cavities have an important net heating effect on the cluster gas. This result can be understood because the simulated cavities were inflated with ultra-hot (but non-relativistic) gas. As these cavities are initially inflated, they perform an amount of heating $\sim P V$ on the surrounding cluster gas, but the thermal energy inside the cavities also contributes to the cluster thermal energy budget. When we perform calculations in which cavities are inflated with ultra-hot gas, we also find a (small) net heating when the total cluster thermal energy includes the energy injected inside the cavity. In this case the total energy introduced by the cavity is $[\gamma /(\gamma-1)] P V=(2.5-4) P V$, depending on the assumed value of $\gamma$ inside the cavity. (After times $\gtrsim 10^{8}$ yr most of the energy of hot-gas cavities is stored in the potential energy of the cluster.) The approximate values of $4 P V$ plotted in Figure 4 show the estimated cavity heating when cavities are formed with ultra-hot gas (e.g. Birzan et al. 2004; Rafferty et al. 2007; McNamara \& Nulsen 2007).

But ultra-hot gas is not an appropriate substitute for cosmic rays. When the diffusion $\kappa$ is sufficiently small and cosmic rays are strongly trapped within the cavities, we find here that the expanding cavities also heat the gas by $\sim P V$. This can be seen in Figure 4 where $\Delta E_{t h}\left(t_{c a v}\right) \approx P V$. But the cosmic ray contents of our cavities never contribute to the cluster thermal energy. Instead, the displacement of cluster gas by the diffusing, buoyant cosmic rays results in an overall expansion and cooling of the cluster gas.

It should also be recognized that the introduction of ultra-hot gas in previous numerical simulations of cavity evolution implicitly suggests that mass as well as cosmic ray energy is transported out from the central AGN, i.e. this conventional means of cavity formation must be regarded as a (mass) circulation flow similar to those we have considered (Mathews et al. 2003; 2004). This assumed non-relativistic mass outflow can be quite large as we discussed in the Introduction. While we assume here that the AGN produces jets of pure relativistic energy, it 
is possible that real jets do in fact carry non-relativistic mass away from the central AGN (as in Brighenti \& Mathews 2006). Nevertheless, current X-ray observations have been unable to detect thermal emission from ultra-hot gas in the cavities (e.g. McNamara \& Nulsen 2007, but see Mazzotta et al. 2002).

\section{CONCLUSIONS}

It is generally agreed that the dominant source of feedback energy in galaxy clusters is accretion onto central black holes (AGNs). Some of this accretion energy is thought to be transported out into the cluster gas by jets, forming X-ray cavities. In previous numerical simulations of X-ray cavity evolution the cavities were formed by introducing ultra-hot, non-relativistic gas at some radius in the cluster gas.

For the first time we consider cavities that are formed exclusively by cosmic rays that can both diffuse through the cluster gas and be advected by it. We discuss the energetics of X-ray cavity and radio lobe evolution in the hot gas within the Virgo galaxy cluster. For simplicity we consider cavities that are formed in time $t_{\text {cav }}=2 \times 10^{7}$ yrs by cosmic rays with total energy $E_{\text {cav }}=10^{58}$ ergs but which have a wide range of cosmic ray diffusion coefficients $\kappa$ since this is currently the most uncertain parameter in cavity-lobe evolution. For simplicity, we do not consider here secular energy losses in the cosmic rays due to synchrotron emission, Coulomb heating, inverse Compton losses, spallation etc., so the total cosmic ray energy can only change due to local expansion or compression by the thermal gas. In this approximation cosmic ray pressure gradients interact with the cluster gas in a perfectly adiabatic fashion.

We conclude that:

(1) As cavities expand during formation, they generate weak shock waves that propagate into the surrounding cluster gas where the shock energy is dissipated. When the cosmic ray diffusion $\kappa$ is low, the maximum shock heating $\sim P V \sim E_{\text {cav }} / 4$ is approached. As $\kappa$ increases the cosmic ray pressure gradients are lower and the shock heating is considerably less. In general, $4 P V$, which varies with time, is an inaccurate measure of $E_{\text {cav }}$. These results are consistent with our earlier studies of $1 \mathrm{D}$ cavity evolution in a uniform gas (Mathews \& Brighenti 2007a).

(2) The longevity of visible X-ray cavities and the radial distance that they move out in the cluster gas during their lifetimes both increase as the cosmic ray diffusion $\kappa$ decreases. Unlike cavities formed with hot gas (e.g. Gardini 2007), cosmic ray cavities remain coherent for $\gtrsim 10^{8}$ yrs.

(3) In spite of shock heating, cavities formed by cosmic rays have a net cooling effect on the cluster gas. This is unlike the cluster heating when cavities are inflated largely with ultra-hot but non-relativistic gas, as commonly assumed, that later contributes to the cluster thermal energy.

(4) The qualitative character of post-cavity energetics remains similar when the cosmic ray diffusion coefficient $\kappa$ varies over a wide range. After several $10^{8}$ yrs the total energy change in the cluster $\Delta E_{\text {pot }}+\Delta E_{t h}+E_{k i n}$ increases as $\kappa$ decreases.

(5) As cosmic rays displace cluster gas, the entire cluster atmosphere expands and cools, increasing the cluster potential energy and decreasing its thermal energy. Successive cavities will result in an irreversible expansion of the cluster gas as long as most of the cosmic rays remain trapped within or near the cooling radius, as in our computed post-cavity evolutions.

(6) Inhomogeneously distributed cosmic rays are an important source of buoyancy and mass flow in the cluster gas. As the cluster gas surrounding cavities is penetrated by diffusing cosmic rays, this gas rises in the cluster potential, driving a net mass outflow in the cluster gas. Radial mass circulation occurs even when no gas flows out in the AGN jets.

(7) After buoyant gas containing cosmic rays flows from the cluster core into distant, low-density regions, it remains there never to return during times comparable to the cluster age. Because of the necessarily positive entropy gradient in the pre-cavity cluster, the entropy in the post-buoyant gas in these distant regions is slightly lower than elsewhere at the same cluster radius.

(8) To quantify the previous conclusion, suppose a mass $\Delta M_{R}$ within the cluster radius $R$ where the X-ray cavity forms is irrevocably transported by cosmic ray buoyancy to distant regions in the cluster gas. We find that a cosmic ray energy of $e_{\odot}=E_{\text {cav }} / \Delta M_{R} \approx 10^{50}$ ergs removes a solar mass of gas from the cavity site. For example in the Virgo cluster a cosmic ray luminosity of only $L_{c r}=e_{\odot} \dot{M}=2 \times 10^{43} \mathrm{erg} \mathrm{s}^{-1}$ can remove gas at the cooling flow rate $\dot{M} \approx 8 M_{\odot} \mathrm{yr}^{-1}$ that would otherwise occur in the absence of feedback energy. Only a fraction $\epsilon_{c r} \approx 5 \times 10^{-4}$ of the accretion energy onto the central black hole in M87/Virgo, $L_{a c c} \approx 0.1 \dot{M} c^{2}$, if converted to cosmic rays that inflate $\mathrm{X}$-ray cavities, is in principle sufficient to shut down the cooling flow.

Our conclusions suggest that cosmic ray buoyancy provides an important new means of understanding or possibly solving the cooling flow problem with an outward mass circulation that may balance the inexorable inflow due to radiation losses. This outward mass flow due to cosmic ray buoyancy has the added attraction of not heating the gas too much - in fact cavity formation with cosmic rays produces a net cooling.

It remains to be determined if the radio emission or $\pi^{0}$ gamma emission from galaxy clusters are at levels that are consistent with the buoyant activity required to arrest the cooling flow rate. After a few $10^{8}$ years most of the cosmic rays will be in very low density gas where such emission will be greatly reduced.

Studies of the evolution of hot cluster gas at UC Santa Cruz are supported by NASA and NSF grants for which we are very grateful.

\section{REFERENCES}

Birzan, L, Rafferty, D. A., McNamara, B. R., Wise, M. W., \& Nulsen, P. E. J. 2004, ApJ, 607, 800
Böhringer, H. \& Morfill, G. E. 1988, ApJ, 330, 609

Brighenti, F. \& Mathews, W. G. 2006, ApJ, 643, 120 
Brighenti, F. \& Mathews, W. G. 2003, ApJ, 587, 580

Brighenti, F. \& Mathews, W. G. 2002, ApJ, 573, 542

Brüggen, M. \& Kaiser, C. R. 2002, Nature, 418, 301

Buote, D. A., Lewis, A. D., Brighenti, F. \& Mathews, W. G. 2003, ApJ, 594, 741

De Grandi, S., Ettori, S., Monghetti, M. \& Molendi, S. 2004, A\&A, 419,7

Drury, L. O. \& Falle, S. A. E. G., 1986, MNRAS, 223, 353

Dunn, R. J. H. \& Fabian, A. C. 2006, MNRAS, 373, 959

Fabian, A. C. et al. 2003, MNRAS, 344, L43

Forman, W. et al. 2007, ApJ, 665, 1057

Forman, W. et al. 2005, ApJ, 635, 894

Fujita, Y., \& Suzuki, T. K. 2005, ApJ, 630, L1

Gardini, A., 2007, A\&A, 464, 143

Ghizzardi, S., Molendi, S., Pizzolato, F. \& De Grandi, S. 2004, ApJ, 609, 638

Govoni, F. \& Feretti, L. 2004, International Journal of Modern Physics D, 13, 1549

Heinz, S., Brug̈gen, M., Young, A. \& Levesque, E. 2006, MNRAS, 373, L65

Jones, T. W. \& Kang, W. 1990, ApJ, 363, 499
Loewenstein, M., Zweibek. E. G. \& Begelman, M. C. 1991, ApJ, 377,392

Mathews, W. G., Faltenbacher, A., \& Brighenti, F. 2006, ApJ, 638, 659

Mathews, W. G. \& Brighenti, F. 2007b, ApJ, (in press) (arXiv:0712.0783)

Mathews, W. G. \& Brighenti, F. 2007a, ApJ, 660, 1137

Mathews, W. G., Brighenti, F. \& Buote, D. A. 2004, ApJ, 615, 662

Mathews, W. G. et al. 2003, ApJ, 596, 159

Mazzotta, P. et al. 2002, ApJ, 567, L37

McNamara, B. R. \& Nulsen, P. E. J. 2007, Annual Review Astronomy \& Astrophysics, 18, 117

Pavlovski, G. et al. 2007, MNRAS (submitted) (arXiv:0709.1790)

Pfrommer, C. \& Ensslin, T. A. 2004, A\&A, 413, 17

Rafferty, D. A., McNamara, B. R., Nulsen, P. E. J., \& Wise, M. W. 2006, ApJ, 652, 216

Reynolds, C. S., McKernan, B., Fabian, A. C., Stone, J. M. \& Vernaleo, J. C., 2005, MNRAS, 357, 242

Stone, J. M. \& Norman, M. L. 1992, ApJS, 80, 753

Temi, P., Brighenti, F. \& Mathews, W. G. 2007b, ApJ, 666, 222

Temi, P., Brighenti, F. \& Mathews, W. G. 2007a, ApJ, 660, 1215 


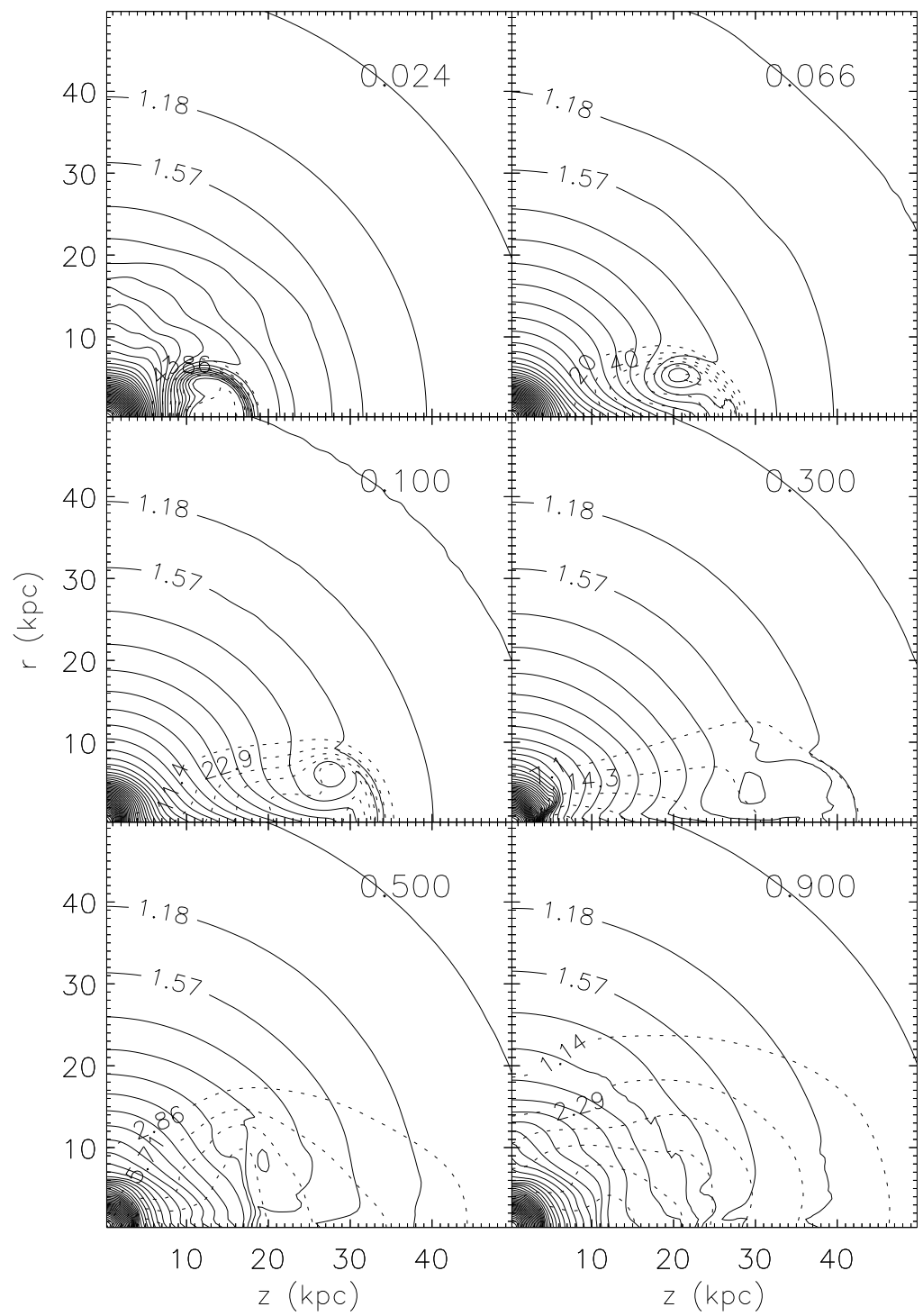

FIG. 1. - Evolution of an X-ray cavity in Virgo with cosmic ray diffusion parameter $n_{e 0}=6 \times 10^{-3} \mathrm{~cm}^{-3}$ at six times shown in Gyrs at the upper right of each panel. Solid lines show the gas density contours $\rho(r, z)$ in units of $10^{-26} \mathrm{~g} \mathrm{~cm}^{-3}$. Dotted lines show with six contours the cosmic ray energy density $e_{c}(r, z)$ in units of $10^{-12} \mathrm{erg} \mathrm{cm}^{-3}$. Two adjacent contours are labeled and others can be found by extending the same additive variation. 


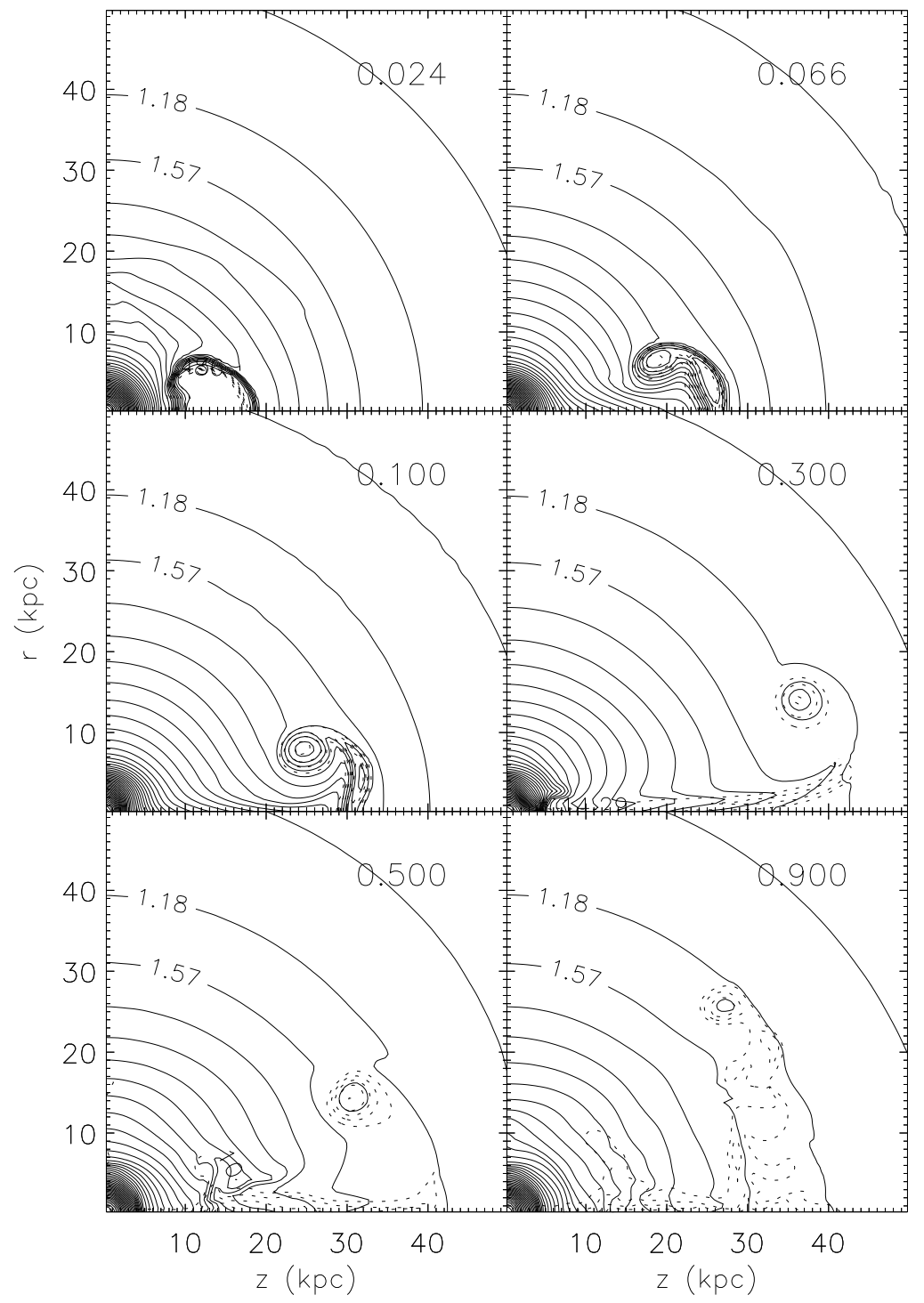

Fig. 2.- Evolution of an X-ray cavity in Virgo with cosmic ray diffusion parameter $n_{e 0}=6 \times 10^{-6} \mathrm{~cm}^{-3}$ at six times shown in Gyrs at the upper right of each panel. Solid lines show the gas density contours $\rho(r, z)$ in units of $10^{-26} \mathrm{~g} \mathrm{~cm}^{-3}$. Dotted lines show with six contours the cosmic ray energy density $e_{c}(r, z)$ in units of $10^{-12} \mathrm{erg} \mathrm{cm}^{-3}$. Two adjacent contours are labeled and others can be found by extending the same additive variation. Due to crowding at early times the cosmic ray contours are difficult to distinguish in the first three panels. For the final three panels the outer two cosmic ray contours are $14.29 \& 31.43$ (panel 0.300), 10.0 \& 20.0 (panel 0.500), and 4.29 \& 11.43 (panel 0.900). 


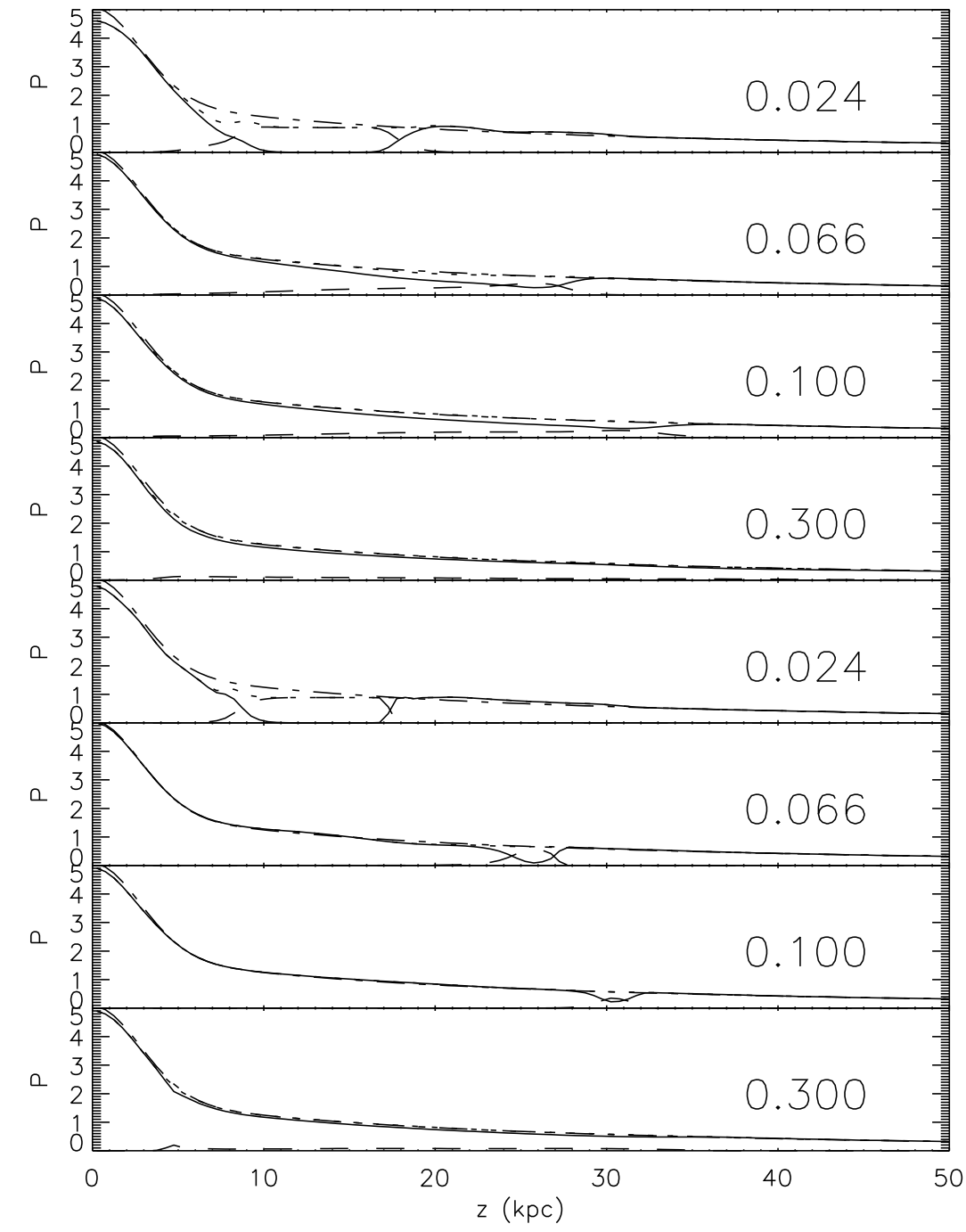

Fig. 3. - Pressure profiles along the $z$-axis in units of $10^{-10}$ dynes $\mathrm{cm}^{-2}$. Each panel contains four (often overlapping) profiles: the gas pressure $P(r)$ (solid lines), cosmic ray pressure $P_{c}(r)$ (long dashed lines), the total pressure $P+P_{c}$ (dotted lines), and the initial gas pressure in the cluster before the cavity is introduced (dash-dotted lines). Upper four panels: show pressure profiles at four times for the evolution in Fig. 1 with $n_{e 0}=6 \times 10^{-3} \mathrm{~cm}^{-3}$. Lower four panels: show pressure profiles at four times for the evolution in Fig. 2 with $n_{e 0}=6 \times 10^{-6} \mathrm{~cm}^{-3}$. Each panel is labeled with the time in Gyrs. 


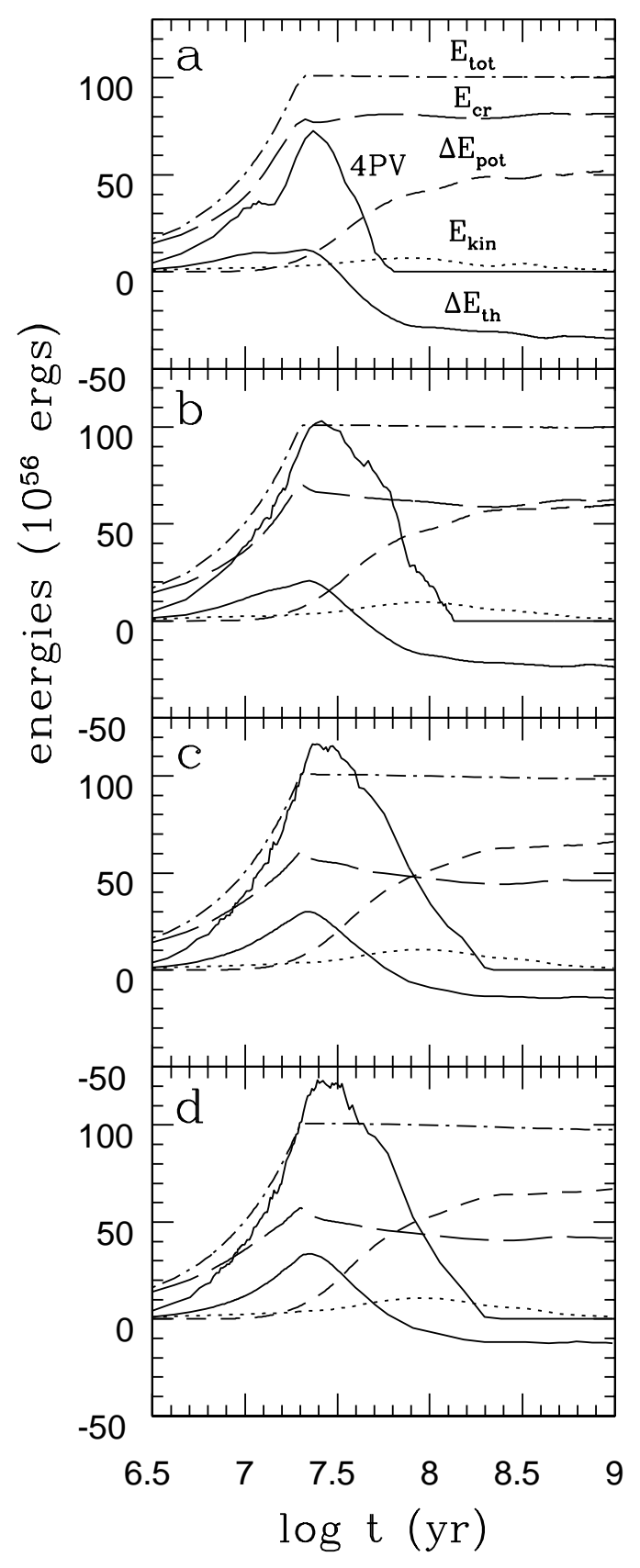

FIG. 4.- Global energy evolution in four cavities with decreasing cosmic ray diffusion coefficient $\kappa \propto n_{e 0}$ characterized by density parameters $n_{e 0}: 6 \times 10^{-3}$ (panel $a$ ), $6 \times 10^{-4}$ (panel $b$ ), $6 \times 10^{-5}$ (panel $c$ ), and $6 \times 10^{-6}$ (panel $d$ ). The energies are labeled as follows: cosmic ray energy $E_{c r}$ (long dashed lines); change in potential energy $\Delta E_{\text {pot }}$ (short dashed lines); kinetic energy $E_{k i n}$ (dotted lines); change in thermal energy $\Delta E_{t h}$ (lower solid lines); and the total energy $E_{t o t}$ (dash-dotted lines). The total energy associated with the approximate cavity volume $4 P V$ is shown in the upper solid lines. All energies are in units of $10^{56}$ ergs and are those in the hemisphere containing our computational grid. 


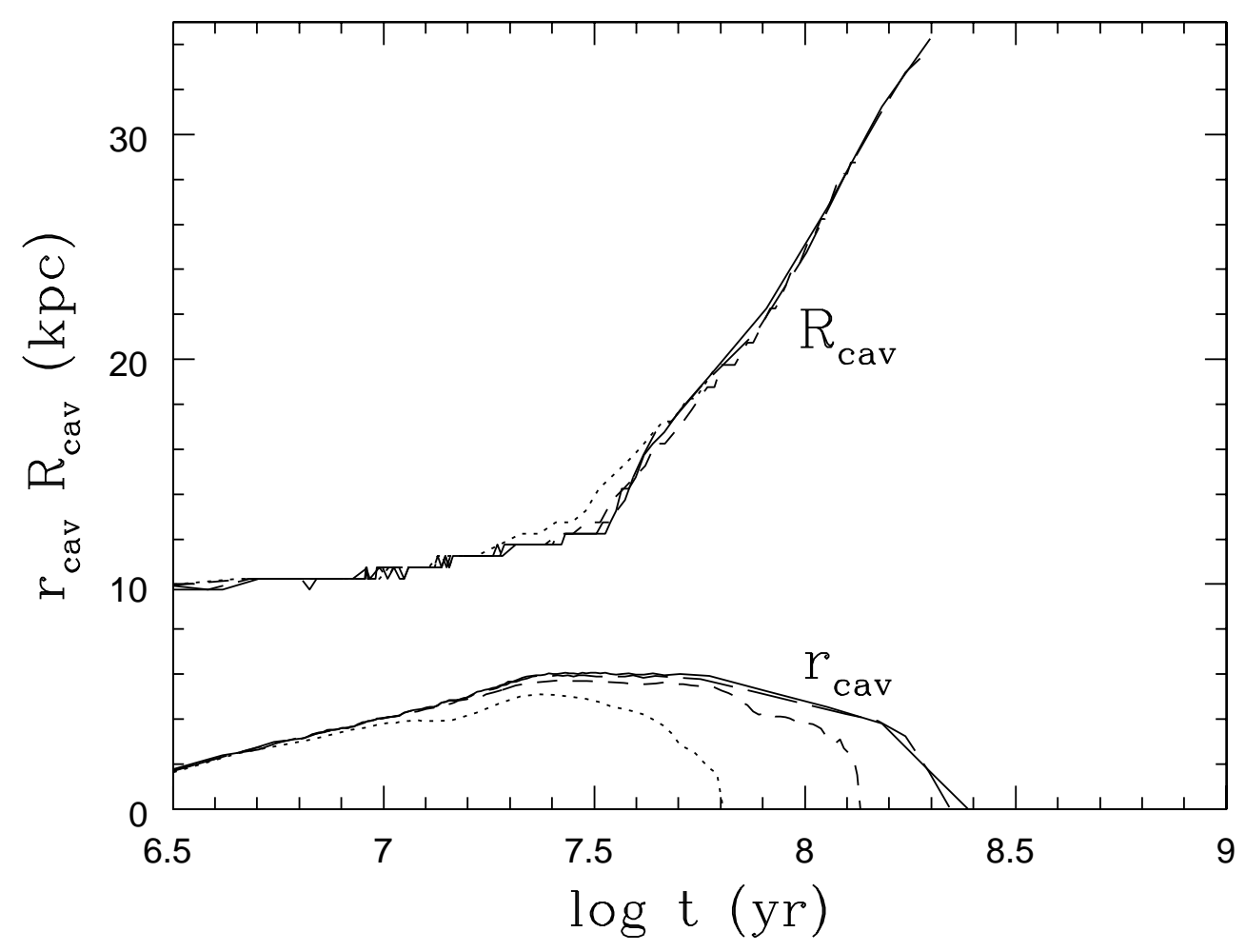

FIG. 5. - Approximate evolution of the cavity radius $r_{c a v}(t)$ and its mean radius in the cluster $R_{\text {cav }}(t)$. The cavity radius is found from the estimated cavity volume $V$ by assuming that the cavity is spherical, $r_{c a v}=[3 V / 4 \pi]^{1 / 3}$. The four lines correspond to the four decreasing cosmic ray diffusivities $\kappa\left(n_{e}, n_{e 0}\right)$ in each panel of Figure $4: a$, dotted line; $b$, short dashed line; $c$, long dashed line; $d$, solid line. Each overlapping trajectory $R_{\text {cav }}(t)$ ends at the time when $r_{\text {cav }} \rightarrow 0$. 


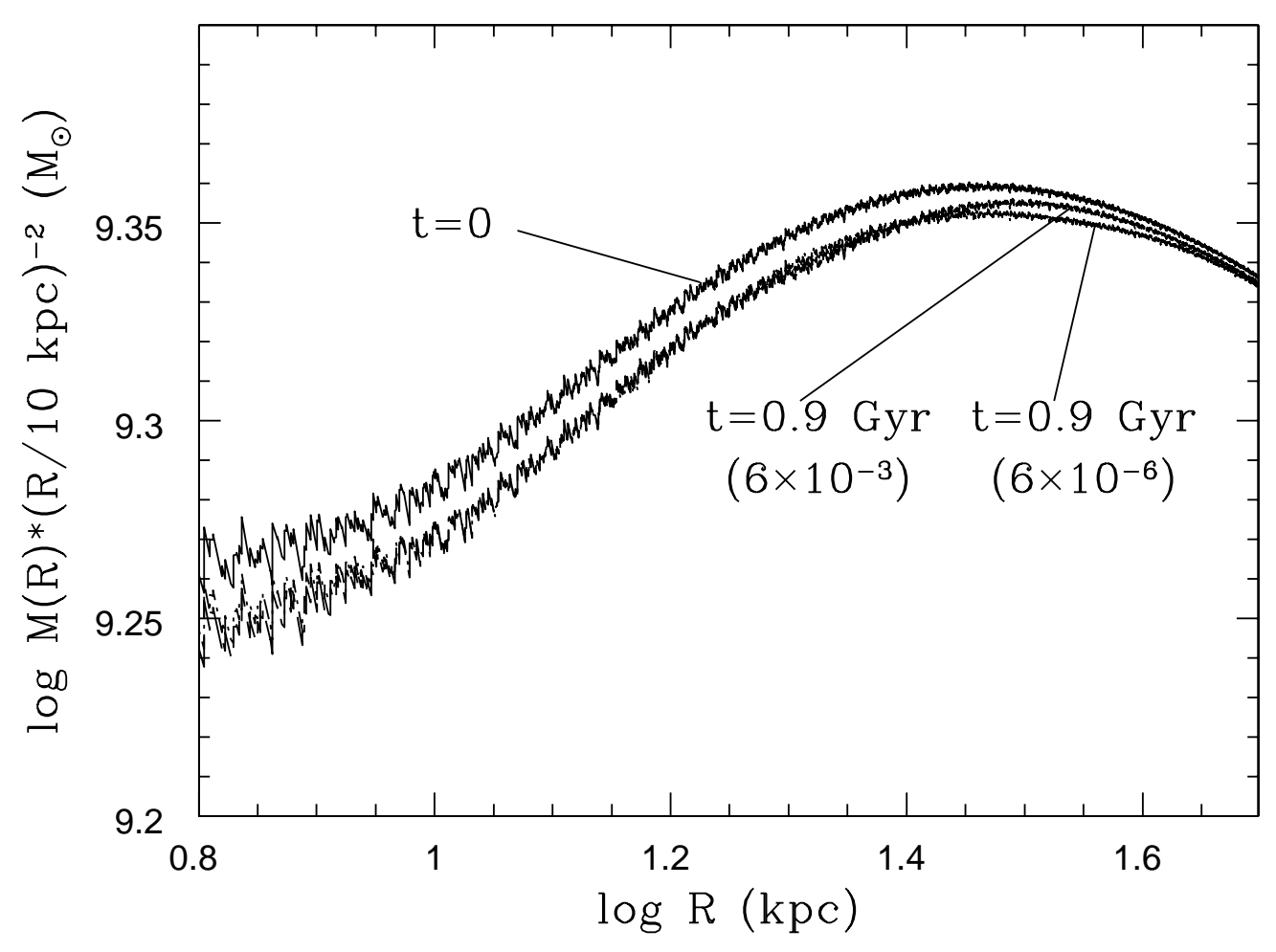

Fig. 6. - Variation of the cumulative spherical mass gas distribution $M(R)$ in one hemisphere of the cluster at times $t=0$ and 0.9 Gyr for two values of the cosmic ray diffusion parameter $n_{e 0}$ shown in parentheses. The cumulative mass is multiplied by $(R / 10 \mathrm{kpc})^{-2}$ to remove most of the radial variation between $R=6.3$ and $50 \mathrm{kpc}$, the approximate Virgo cooling radius. 


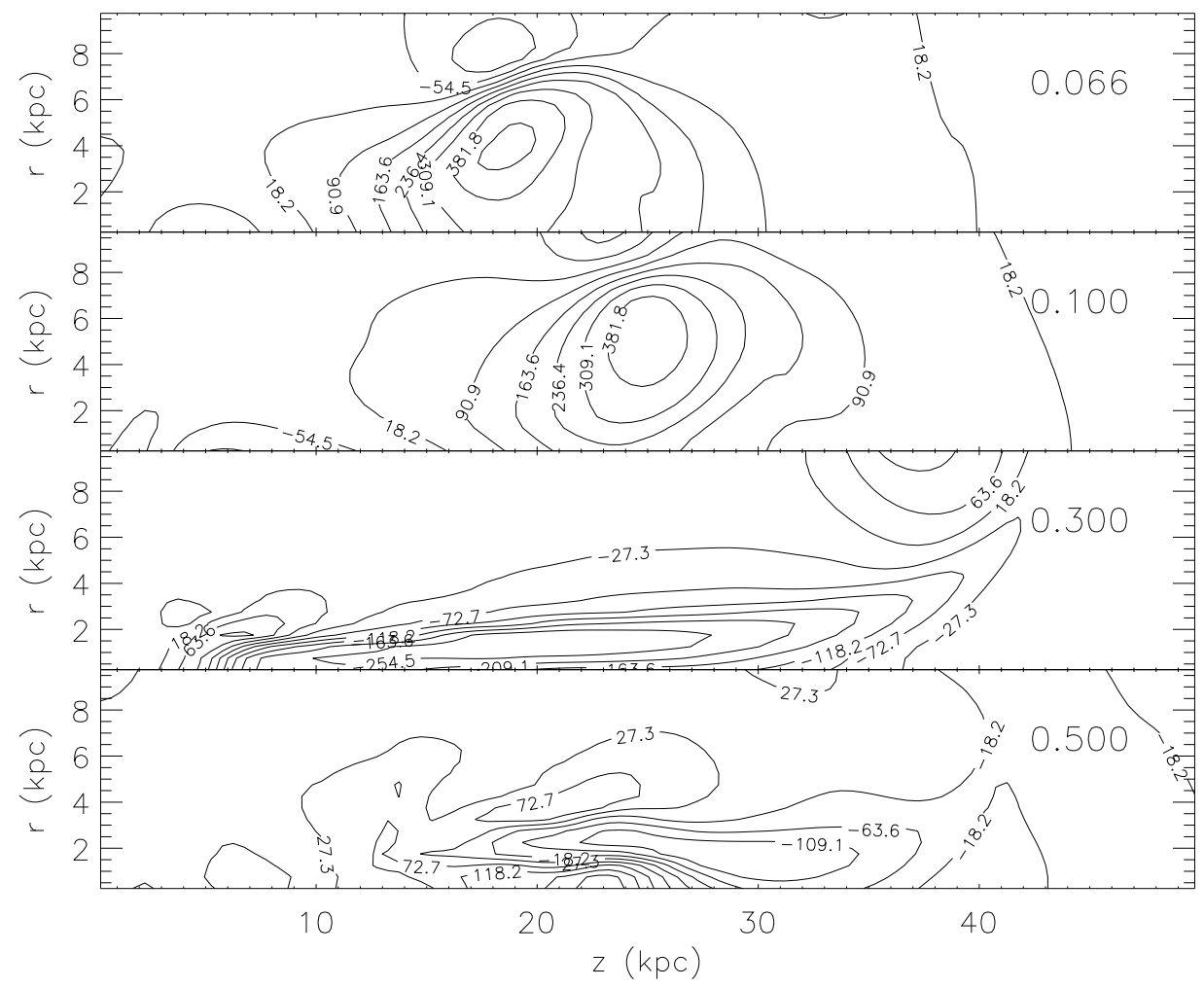

FIG. 7. - Evolution of the radial velocity $v_{r}(r, z)$ near the $z$-axis with cosmic ray diffusion parameter $n_{e 0}=6 \times 10^{-6} \mathrm{~cm}^{-3}$ at four times shown in Gyrs at the upper right of each panel. Contours are labeled with values of $v_{r}$ in $\mathrm{km} \mathrm{s}^{-1}$. 
FIG. 8. - Top panel: Correlation of the cosmic ray partial pressure $P_{c} /\left(P+P_{c}\right)$ with the entropy change $\Delta s$ in each computational zone for the low- $\kappa$ post-cavity flow over time $t=0.9$ Gyrs. Each point represents a computational zone within cluster radius $R=50 \mathrm{kpc}$. Bottom panel: Location of regions in the cluster with high cosmic ray partial pressures $\left(P_{c} /(P+P c)>0.3\right)$ (points) and regions of large negative entropy change $(\Delta s<-0.1)$ (open squares). 
This figure "f8.gif" is available in "gif" format from: http://arxiv.org/ps/0805.2441v1 